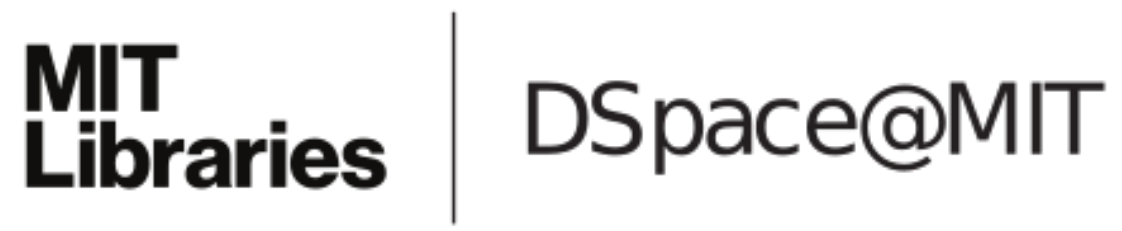

\author{
MIT Open Access Articles
}

A pH-responsive supramolecular polymer gel as an enteric elastomer for use in gastric devices

The MIT Faculty has made this article openly available. Please share how this access benefits you. Your story matters.

Citation: Zhang, Shiyi; Bellinger, Andrew M.; Glettig, Dean L.; Barman, Ross; Lee, Young-Ah Lucy; Zhu, Jiahua; Cleveland, Cody et al. "A pH-Responsive Supramolecular Polymer Gel as an Enteric Elastomer for Use in Gastric Devices." Nature Materials 14, no. 10 (July 2015): 10651071 @ 2014 Macmillan Publishers Limited, part of Springer Nature

As Published: http://dx.doi.org/10.1038/nmat4355

Publisher: Nature Publishing Group

Persistent URL: http://hdl.handle.net/1721.1/109409

Version: Author's final manuscript: final author's manuscript post peer review, without publisher's formatting or copy editing

Terms of Use: Article is made available in accordance with the publisher's policy and may be subject to US copyright law. Please refer to the publisher's site for terms of use. 
Published in final edited form as:

Nat Mater. 2015 October ; 14(10): 1065-1071. doi:10.1038/nmat4355.

\title{
pH-responsive supramolecular polymer gel as an enteric elastomer for use in gastric devices
}

\author{
Shiyi Zhang ${ }^{a}$, Andrew M. Bellinger ${ }^{a, b}$, Dean L. Glettiga ${ }^{a}$, Ross Barman ${ }^{a, c}$, Young-Ah Lucy \\ Lee $^{\mathrm{a}}$, Jiahua Zhu ${ }^{\mathrm{d}}$, Cody Cleveland ${ }^{\mathrm{a}}$, Veronica A Montgomery ${ }^{\mathrm{a}}$, Li Gu${ }^{\mathrm{a}}$, Landon D. Nash ${ }^{\mathrm{e}}$,

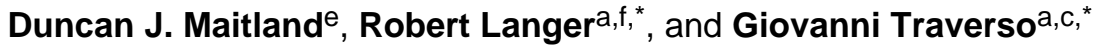 \\ aDepartment of Chemical Engineering and Koch Institute for Integrative Cancer Research, \\ Massachusetts Institute of Technology, Cambridge, MA 02139 \\ ${ }^{b}$ Cardiovascular Division, Department of Medicine, Brigham and Women's Hospital, Harvard \\ Medical School, Boston, MA 02115 \\ 'Division of Gastroenterology, Massachusetts General Hospital, Harvard Medical School, Boston, \\ MA 02114 \\ ${ }^{d}$ Center for Nanophase Materials Sciences, Oak Ridge National Laboratory, Oak Ridge, TN \\ 37831 \\ eDepartment of Biomedical Engineering, Biomedical Device Laboratory, Texas A\&M University, \\ College Station, TX 77843 \\ fHarvard-MIT Division of Health Sciences and Technology, Massachusetts Institute of \\ Technology, Cambridge, MA 02139
}

\section{Abstract}

Devices resident in the stomach -- which are used for a variety of clinical applications including nutritional modulation for bariatrics, ingestible electronics for diagnosis and monitoring, and gastric retentive dosage forms for prolonged drug delivery -- typically incorporate elastic polymers to compress the devices during delivery through the esophagus and other narrow orifices in the digestive system. However, in the event of accidental device fracture or migration, the nondegradable nature of these materials risks intestinal obstruction. Here, we show that an elastic, pHresponsive supramolecular gel remains stable and elastic in the acidic environment of the stomach but can be dissolved in the neutral-pH environment of the small and large intestines. In a large

Users may view, print, copy, and download text and data-mine the content in such documents, for the purposes of academic research, subject always to the full Conditions of use:http://www.nature.com/authors/editorial_policies/license.html\#terms

”To whom correspondence may be addressed. ctraverso@partners.org or rlanger@mit.edu.

Author contributions

S.Z., R.S.L. and G.T. designed the material and experiments. S.Z. prepared the material and the device. S.Z., A.M.B., D.L.G., R.B., Y.-A.L.L., J.Z., V.A.M., C.C., L.D.N., D.J.M., L.G. and G.T. characterized the material, analyzed the data and wrote the paper. R.S.L. and G.T. supervised the research. All authors discussed the progress of research and reviewed the manuscript.

Additional information

Supplementary information is available in the online version of the paper. Reprints and permissions information is available online at www.nature.com/reprints. Correspondence and requests for materials should be addressed to R.S.L. or G.T.

Competing financial interests

The authors declare Provisional US patent application No. 62/010,992 filed on June 11, 2014. 
animal model, prototype devices with these materials as the key component demonstrated prolonged gastric retention and safe passage. These enteric elastomers should increase the safety profile for a wide range of gastric retentive devices.

Interest in the development of gastric-resident and gastric-retentive devices has been increasing due to their broad applications including: bariatric interventions for nutritional modulation to address the global obesity epidemic, ${ }^{1-3}$ ingestible electronics for real time physiological monitoring and improving patient health, ${ }^{4-7}$ and daily dosage forms for prolonged oral drug deliveries. ${ }^{8-12}$ To achieve prolonged retention in the gastric cavity without exiting through the pylorus (diameter $\sim 1.3 \mathrm{~cm}$ ), ${ }^{13,14}$ gastric devices are often designed to expand to greater than $2 \mathrm{~cm}$ in diameter. At the same time, to ensure the safe delivery of large objects through the narrow esophagus (diameter 1.5-2 cm), ${ }^{15}$ those gastric devices are often made of, at least in part, elastic polymers for compacting or folding whole devices into smaller configurations. ${ }^{16}$ Unfortunately, due to the non-degradable or nondissociable nature of elastic polymers, those large-sized devices can cause complications, such as intestinal obstruction after migration of fractured components or even whole devices, requiring surgical intervention for removal. ${ }^{17-21}$ These complications have been observed across a range of devices including ingestible electronic devices, ${ }^{18}$ percutaneous feeding tubes ${ }^{19}$ as well as intragastric balloons for weight loss. ${ }^{20,21}$ In spite of the broad and increasing clinical utility of these devices, there is one striking omission in their functions a mechanism to prevent intestinal obstruction upon exiting the stomach and a significant need has been established for such materials given their potential application for extended retention systems. ${ }^{22}$

Given the significant $\mathrm{pH}$ difference between the gastric $(\mathrm{pH} 1-3)$ and intestinal $(\mathrm{pH} \sim 6.8$ ) environments, we envision a $\mathrm{pH}$ responsive elastomer that is stable in acidic condition but dissolvable in neutral or alkaline conditions may address this unmet clinical need. Enteric polymers have been previously developed and are generally used as coatings of oral pills and capsules to protect the active pharmaceutical ingredients from the high acidity in the gastric environment. ${ }^{23,24}$ These materials share a common structure by having a large hydrophobic moiety and carboxyl groups for $\mathrm{pH}$ responsiveness. Existing enteric polymers are generally rigid and often brittle and therefore have not found utility in the application of gastric-retentive devices so far. Combining elastic and enteric properties remains a great challenge for material development. The recent advances in supramolecular polymer gels, ${ }^{25,26}$ present many examples of materials with tunable mechanical properties and various environmental stimuli-responsiveness. ${ }^{27-31}$ For instance, a family of supramolecular polymer gels that is loosely cross-linked by static interactions, and possesses good elasticity and stimuli-responsiveness to $\mathrm{NaCl}$ solution which in turn disrupts the charge-charge interactions has been described. ${ }^{32} \_$ENREF_28 Though studies on supramolecular polymer gels and responsive polymers have enriched our knowledge towards polymeric materials, only a few applications taking advantage of their unique properties have been demonstrated. ${ }^{33,34}$ We hypothesized that a supramolecular polymer gel, which is physically cross-linked by hydrogen bonds between carboxyl groups, could have good elasticity and also be enteric for the next generation gastric devices enabling dissolution of devices into components that can pass through the gastrointestinal tract. 
Here we describe, to the best of our knowledge, the first material combining both elastic and enteric properties, allowing the construction of gastric devices with facile delivery, prolonged gastric retention, and an improved safety profile. The material is a unique supramolecular polymer gel with elastic properties in acidic environments and which dissolves in water under neutral conditions. Using this enteric elastomer (EE) as the key component, we built a variety of prototype gastric-resident devices, which showed prolonged gastric retention (2-7 days) after delivery via administration of an encapsulated device or endoscopic placement of the device in the stomach, with subsequent safe passage through the lower gastrointestinal tract upon dissociation in a large animal model. Although there have been extensive studies and commercial examples of gastric retentive devices since the 1970s, ${ }^{9,35}$ this manuscript presents the first demonstration of both extremely prolonged gastric retention and safe gastrointestinal passage with the help of this innovative combination of elastic and enteric properties contained in one material.

\section{Preparation of polymer gel as enteric elastomer (EE)}

Fig. 1 depicts the proposed supramolecular network structure of the EE polymer gel. The EE consists of two synthetic macromolecules, poly(acryloyl-6-aminocaproic acid) (PA6ACA, Scheme S1) and poly(methacrylic acid-co-ethyl acrylate) (EUDRAGIT® L100-55). L100-55 is a pharmaceutical grade enteric polymer from Evonik Industries. PA6ACA, synthesized by a previously reported method, ${ }^{36}$ is structurally similar to traditional enteric polymers (e.g. L 100-55, cellulose acetate succinate and hydroxyl propyl methyl cellulose phthalate). PA6ACA has side chains of sufficient length for the terminal carboxyl groups to be flexible and accessible allowing the formation of intermolecular hydrogen bonds as shown in Fig. 1a. ${ }^{36}$ In the acidic environment when carboxyl groups are not deprotonated, inter-chain hydrogen bonds between carboxyl groups and amide units on PA6ACA and L100-55 provide a loosely cross-linked supramolecular network with water trapped inside that contributes to the elastic property of the materials. In neutral or alkali aqueous environments, the carboxyl groups are deprotonated, eliminating the inter-molecular hydrogen bonds, resulting in rapid dissolution.

EEs with various compositions and properties were synthesized by co-precipitation of a solution of PA6ACA sodium salt and L 100-55 sodium salt in polymer weight ratios of 1:0, $1: 1$, and $1: 2$ with the addition of $6 \mathrm{M} \mathrm{HCl}$ solution and compacting by ultracentrifugation (Fig. 1b, see Methods for details). The co-precipitation and ultracentrifugation process yielded macroscopically homogeneous materials with tough elastic properties and relatively low water contents ( $<35 \%$, measurement method in supporting information). Fig. 1c shows a typical piece of EE taken from the ultracentrifuge tube (PA6ACA:L100-55 1:2 shown here). EE could be easily cut into various shapes for the construction of devices or mechanical characterizations. In preliminary mechanical testing (PA6ACA:L100-55 1:2 as pictured in Fig. 1d), a cuboid-shape was pulled to three times its original length and fully recovered its shape 5 minutes after the external force was removed, demonstrating the desired elastic property without material fatigue. 


\section{Physical characterization of EE}

To better understand the structure-property relationship of EEs with various PA6ACA to L100-55 ratios, we characterized the nanostructure, morphology, cytotoxicity, swelling, mechanical and enteric properties of these materials. At the molecular level, the hydrogenbonding network of EEs was characterized by using small angle X-ray scattering (SAXS) and infrared (IR) spectroscopy. The scattering profile of PA6ACA gel (red curve in Fig. 2a) presents four broad peaks. Two peaks were founds in the higher q-region corresponding to periodic distances of around $3.1 \AA$ and $2.3 \AA$ which were also found in pure water and thus can be attributed to hydrogen-bonding (defined as type IV) between $\mathrm{H}_{2} \mathrm{O}$ molecules in the gel. ${ }^{37}$ The other two peaks in the lower q-region of SAXS profile represent two distinct periodic distances of $12.5 \AA$ and $5.7 \AA$, which can be assigned to two co-existing hydrogenbonding configurations between PA6ACA molecules in the gel, the face-on configuration (type I) and the interleaved configuration (type III), respectively (Fig. 2b). The formation of the two PA6ACA hydrogen-bonding configurations was further supported by IR spectroscopy (Fig. S1). ${ }^{36}$ When blending PA6ACA with L100-55 in the gel, a new peak appears in the intermediate q-region ( $6.3 \AA$ ) of SAXS profile, suggesting the formation of a new hydrogen-bonding configuration (type II, Fig. 2b) between PA6ACA and L100-55. Increasing the content of L100-55 in the polymer gels results in a relative increase in peak (II) with reduction of peaks (I) and (III) in the SAXS profiles. Scanning electron microscopy (SEM) was employed to study the microstructure of EEs. As revealed by SEM images of lyophilized gels (Fig. 2c) of three formulations of EE demonstrated porosity in the micrometer range with higher blending ratio of L100-55 correlating with decreasing pore size. The water content decreased from $31.6 \pm 3.8 \%$ in PA6ACA itself, to $27.7 \pm 4.6 \%$ in the EE with ratio 1:1, and to $26.4 \pm 3.5 \%$ in the $\mathrm{EE}$ with ratio $1: 2$, which is consistent with the SEM porosity findings.

We further tested the elastic and enteric properties, which are the two key functions of the EEs. The mechanical properties and the way in which these are influenced by the blending ratio of PA6ACA to L100-55 were studied using immersion tensile-stress tester in SGF at $37^{\circ} \mathrm{C}$. With increasing amount of L100-55, the Young's modulus and tensile strength increased, while the strain decreased from $1207 \%$ strain in pure PA6ACA and 1230\% strain in the $\mathrm{EE}$ with the ratio $1: 1$ to $943 \%$ in the EE with the ratio 1:2 (Fig. 2d). The stress-strain test suggests that mechanical properties of EEs can be engineered by tuning the blending ratio of PA6ACA and L100-55. The $\mathrm{pH}$-dependent dissolution properties of EEs were evaluated in simulated gastric fluid ( $\mathrm{SGF}, \mathrm{pH}=\sim 1.2$ ) and simulated intestinal fluid (SIF, $\mathrm{pH}=\sim 6.8$ ). As shown in Fig. 2e, all three formulations of EEs showed long-term stability in SGF without detectable mass loss over 4 days. In contrast, within the same period of time, all three EEs were nearly dissolved in SIF in a pseudo-zero order manner with similar dissolution rates. To further modulate the enteric properties of EEs, we synthesized a copolymer of $\mathrm{N}$-acryloyl 6-aminocaproic acid (A6ACA) and the more hydrophobic monomer N-acryloyl 11-aminoundecanoic acid (A11AUA) creating P(A6ACA $0.5^{-}$ coA11 $\mathrm{AUA}_{0.5}$ ) (Scheme S1, $\left.M_{n}=82,300-170,600 . M_{w}=358,400-655,900\right)$. This copolymer was blended with L100-55 at a weight ratio of 1:2 resulting in a material that completely dissolved in SIF in 18 days (Fig. S2). Therefore, modulating polymer gel 
compositions by physical blending or chemical copolymerization, both elastic and/or enteric properties of EEs could be adjusted.

To evaluate the biocompatibility and biosafety of EEs, EE sodium salt forms were tested for their cytotoxicity towards multiple cell lines, including HeLa, HEK293 and the intestinal lines Caco-2 (C2BBe1 clone) and HT29-MTX-E12 (Fig. S3). No significant cytotoxicity was observed for all three formulations of EEs over a wide range of concentrations from $0.078-20 \mathrm{mg} / \mathrm{mL}$ at the end of a $72 \mathrm{~h}$ incubation period. The observed cytotoxicity at very high concentrations ( $\mathrm{LD}_{50}$ above $4.71 \mathrm{mg} / \mathrm{mL}$ ) may be due to changes in $\mathrm{pH}$ or viscosity of cell culture medium after dissolving a large amount of high molecular weight polymer sodium salts. EEs were further evaluated for swelling behavior in several commonly ingested fluids including vegetable oil and ethanol. EEs did not swell and maintained their integrity in acidic aqueous solutions ( $\mathrm{pH} \leq 5.0)$, and in an acidic solution mixed with $10 \mathrm{wt}$ $\%$ vegetable oil (see supporting information). PA6ACA was evaluated for its ability to absorb ethanol. PA6ACA did not swell noticeably in 10\% ethanol (Fig. S4), supporting the compatibility of this family of materials with common components of diets.

\section{Fabrication and testing of gastric retentive devices}

As a step towards the goal of using EEs as key building blocks in gastric-retentive devices, we integrated EE and polycaprolactone (PCL) in prototype gastric-retentive devices. EE with PA6ACA/L100-55 1:2 weight ratio was selected for the fabrication and testing of gastric devices in the rest of this study due to its high tensile strength. For the structural component of the gastric devices we chose PCL, which is widely used as a biomaterial for implants and as a drug carrier due to its proven biocompatibility, excellent mechanical properties and ease in manufacturing. ${ }^{38,39}$ Generally, we first used a 3D printer to generate positive molds for the generation of negative polydimethylsiloxane (PDMS) molds, then placed pieces of EE into the molds and melted $\mathrm{PCL}$ at $70{ }^{\circ} \mathrm{C}$ to interface $\mathrm{PCL}$ with the EE for the formation of the integrated EE-PCL device. To assess the strength and integrity of the joint interface between EE and PCL, we placed EE in the center of a dog-bone shaped device with PCL on both sides and deformed the dog-bone by $180^{\circ}$ as well as linear extension until fracture. As shown in Fig. S5, the EE has a low enough Young's modulus to tolerate $180^{\circ}$ bending. During fracture testing, the EE-to-PCL interfaces were intact, suggesting the stability of the interface and the feasibility of using PCL as a co-building block with EE for the fabrication of gastric resident devices.

To demonstrate the utility of elastic and enteric functions of EE in gastric devices, we fabricated and tested a ring composed of PCL arcs with intervening EE linkers (Fig. 3). The maximal diameter of a device enabling gastric retention by preventing passage through the pylorus has been previously established as a key parameter. ${ }^{40-42}$ Considering that the aperture diameter of the resting human pylorus is $12.8 \pm 7.0 \mathrm{~mm},{ }^{14}$ we prepared a gastric retentive device in a ring-shaped PDMS mold with outer diameter of $32 \mathrm{~mm}$, inner diameter of $28 \mathrm{~mm}$, width of $2 \mathrm{~mm}$, and depth of $2 \mathrm{~mm}$. EE was cut into cuboid sections with the dimensions $6 \mathrm{~mm} \times 4 \mathrm{~mm} \times 2 \mathrm{~mm}$, fitted in the molds and then dried by vacuum. This was followed by PCL placement and melting (Fig. 3a). As shown in Fig. 3b, the resulting ringshaped device can be encapsulated by bending the elastic components up to 180 degrees to 
fit into a standard 000 gelatin capsule. To simulate deployment and retention in the stomach environment, the encapsulated circle-shaped device was placed in SGF at $37^{\circ} \mathrm{C}$. The deployed device escaped from the capsule and recovered its original shape within $8 \mathrm{~min}$ (Fig. 3c). The medium was changed to SIF and the EE linkers slowly swelled and dissolved. As a result, the ring-shaped device gradually disassembled within 12 hours (Fig. 3d). The elastic property of the EE enabled the encapsulation and restoration of the ring-shaped device following release from the capsule, while the enteric property allowed the dissociation of the device in SIF.

\section{In vivo evaluation of gastric-resident devices in a large animal model}

Having established in vitro the elastic and enteric properties imparted by the incorporation of the EE into prototypic devices, we next tested the in vivo application of gastric-retentive devices formed with EE, using a Yorkshire pig animal model. Yorkshire pigs weighing 45-55 kg have gastric and intestinal anatomy and dimensions similar to humans and have been previously used in evaluation of other gastrointestinal devices. ${ }^{43}$ Ring-shaped devices as depicted in Fig. 3 were formed and encapsulated in 000 gelatin capsules with the addition of $1 \mathrm{~mm}$ stainless steel balls within the PCL arms for radiographic monitoring. ${ }^{12}$ Under moderate sedation, the capsule was introduced through the esophagus under endoscopic visualization. The encapsulated ring-shaped device deployed and restored its baseline shape in the stomach within $15 \mathrm{~min}$ (Fig. 4a). Four individual experiments on four different pigs were performed demonstrating gastric retention of the device for 2-5 days (Fig. $4 \mathrm{~b}$ and Fig. 4d). No intact devices were visualized outside of the stomach suggesting device breakage first occurred in the stomach. Loss of the intact device was visualized radiographically where the partial dissolution and/or rupture of one or two EE linkers was noted resulting in linearization of the closed structure enabling easier passage out of the stomach (Fig. S6) ${ }^{41}$. Upon passage out of the stomach the dissolvable EEs disintegrated resulting in the small rigid elements capable of safe passage without evidence of intestinal obstruction (Fig. 4c and Fig. 4e). Throughout the experiments the animals were found to have normal eating and stooling patterns and did not display any signs of gastrointestinal obstruction either clinically or radiographically. Radiographic visualization for the experiments above was enabled by the inclusion of radio-opaque beads in the PCL segments of the devices. To evaluate the possibility that the stainless steel beads in the PCL arms contributed to gastric retention, four encapsulated ring-shaped devices without iron beads were deployed into two pigs (two capsules per animal). Endoscopic imaging was used to evaluate the devices in the gastric cavity at the time points of 0.5 hour, 2 days, 4 days and 7 days post deployment. All four rings were identified and were intact in the stomachs of the 2 animals after 0.5 hours, 2 and 4 days, while only one ring was identified after 7 days (Fig. S7). Gastric retention did not appear to be significantly affected by the elimination of the stainless steel beads which represented $\sim 20 \%$ of the total mass of the device.

The elastic function of the EE enabled the circle-shaped device to be folded into the standard 000 capsule for comfortable oral delivery and also enabled shape recovery for prolonged gastric retention, after dissolution of the capsule. The enteric function permitted the dissociation of the device into small pieces for safe passage through the lower gastrointestinal tract. This prototype device achieved extended gastric retention for 2-7 days, 
when compared to the maximum of 1-2 days of gastric retention achieved by other reported gastric retentive devices delivered by capsules.., 35

Beyond the self-deployable gastric-retentive device delivered by capsules, we also explored exemplary gastric-resident devices for the endoscopic delivery and placement, including large devices composed similarly of PCL rigid segments linked together with EE and that formed the letters "M.I.T.". Those exemplary gastric-resident devices were constructed with EE and PCL embedded with iron beads by using M-, I-, and T-shaped PDMS molds. These shapes could be folded and delivered through the esophagus with endoscopic assistance. Radiographic images show elastic restoration of the M, I, and T-letter shapes in three pig stomachs (Fig. 5 b, c, e, f, h, i) right after delivery. Endoscopic images also confirmed gastric retention of all three letters, and found no obstruction caused by those devices (Fig. 5 $\mathrm{d}, \mathrm{g}, \mathrm{j}$ ). All three M, I, and T-shaped devices were retained in the gastric cavity for 2-5 days before their fragmentation (Fig. S8). EEs can be used in the fabrication of a variety of gastric devices to prevent intestinal obstruction upon exiting the stomach. The incorporation of dissolvable EE linkers enables the development of devices with potentially significantly improved safety profiles by allowing for fragmentation of the device into segments that can easily pass through the gastrointestinal tract. As demonstrated by the M.I.T. experiment, devices of significant size can be safely deployed in the stomach and upon dissolution of the EEs pass safely through the gastrointestinal tract. The restriction on the size of gastric resident devices becomes the ability to deliver such a device to the gastrointestinal tract. In a conscious patient, the delivery of a device is limited by the size of the capsule or tablet that a patient is willing to swallow. Through endoscopic or minimally invasive access techniques, devices incorporating EEs could be introduced of nearly arbitrary size limited by the diameter of the esophagus (in humans $\sim 2 \mathrm{~cm}$ ), but capable of safe fragmentation into small pieces.

\section{Outlook}

In summary, to the best of our knowledge, we have demonstrated the first supramolecular enteric and elastic polymer gel and showed a direct application in the construction of safe gastric retentive devices. The combination of elastic and enteric properties enhances the safety profile of such devices. We envision this material could change the design and radically improve the adoption novel gastric resident devices including those for weight control, ingestible electronics and prolonged drug delivery.

\section{Methods}

Materials

6-Aminocaproic acid, 11-aminoundecanoic acid, $\mathrm{NaOH}$, hydrochloric acid (ACS reagent, $37 \%$ ), $\mathrm{NaCl}$, tetramethylethylenediamine, ammonium persulfate, polycaprolactone (PCL, average Mn 80,000) and KH2PO4 were used as received from Sigma-Aldrich Company (St. Louis, MO). Acryloyl chloride was purchaded from Sigma and vacuum distilled before using. Nanopure water $(18 \mathrm{M} \Omega \bullet \mathrm{cm})$ was acquired by means of a Milli-Q water filtration system, Millipore Corp. (St. Charles, MO). 1L of simulated gastric fluid (SGF, pH 1.2) was made by dissolving $2 \mathrm{~g} \mathrm{NaCl}$ and $8.3 \mathrm{~mL}$ concentrated $\mathrm{HCl}$ in water and adjusting to 
$1000 \mathrm{~mL}$ with water. $1 \mathrm{~L}$ of simulated intestinal fluid (SGF, $\mathrm{pH} \sim 6.8$ ) was made by dissolving $6.8 \mathrm{~g} \mathrm{KH} 2 \mathrm{PO} 4$ and $0.896 \mathrm{~g} \mathrm{NaOH}$ in water and adjusting to $1000 \mathrm{~mL}$ with water.

\section{Synthesis of PA6ACA sodium salt}

To a nitrogen bubbled solution containing $10 \mathrm{~g}$ (54.1 mmol) A6ACA, $2.16 \mathrm{~g}(54.1 \mathrm{mmol})$ $\mathrm{NaOH}$, and $6.3 \mathrm{mg}(0.0541 \mathrm{mmol})$ tetramethylethylenediamine (TMEDA) dissolved in 400 $\mathrm{mL}$ nanopure water at $40{ }^{\circ} \mathrm{C}$ was added a solution of $62 \mathrm{mg}(0.270 \mathrm{mmol})$ ammonium persulfate in $10 \mathrm{~mL}$ nanopure water. The reaction mixture was allowed to stir for $12 \mathrm{~h}$ for the polymerization. The polymer solution was transferred to dialysis tubes (MWCO 3500 Da) for dialysis for three days and lyophilized, obtaining a white solid powder with an average yield of $95 \% .{ }^{1} \mathrm{H}$ NMR ( $\left.\mathrm{D}_{2} \mathrm{O}, \mathrm{ppm}\right): \delta 3.15$ (s, CONHCH2CH2), 2.20 (d, $\mathrm{CH} 2 \mathrm{CH} 2 \mathrm{CH} 2 \mathrm{COO}-$ ), 2.02 (b, CONHCH2CH2), 1.57 (s, CH2CH2CH2COO-), 1.55 (s, $\mathrm{CONHCH} 2 \mathrm{CH} 2), 1.33$ (s, CH2CH2CH2COO-), 1.70-1.25 (b, CH2CHCO). ${ }^{13} \mathrm{C}$ NMR $\left(\mathrm{D}_{2} \mathrm{O}\right.$, ppm): $\delta 183.52,176.13,39.56,37.42,28.33,28.26,26.31,25.51 . M_{n}=61,600-112,700$. $M_{w}=347,300$ - 466,300. IR: $3600-3000,2911,2843,1638,1536,1395,1302,1210,1165$, $1099 \mathrm{~cm}-1$. DSC: $(\mathrm{Tg})=94.2^{\circ} \mathrm{C}$.

\section{Synthesis of $\mathrm{P}\left(\mathrm{A} 6 \mathrm{ACA} \mathrm{A}_{0.5}-\mathrm{co}-\mathrm{A} 11 \mathrm{AU} \mathrm{A}_{0.5}\right)$ sodium salt}

To a nitrogen bubbled solution containing $10 \mathrm{~g}$ (39.2 mmol) A11AUA, $7.25 \mathrm{~g}(39.2 \mathrm{mmol})$ A6ACA, $3.14 \mathrm{~g}(78.4 \mathrm{mmol}) \mathrm{NaOH}$, and $9.1 \mathrm{mg}(0.0784 \mathrm{mmol})$ tetramethylethylenediamine (TMEDA) in $700 \mathrm{~mL}$ nanopure water at $40{ }^{\circ} \mathrm{C}$ was added a solution of $89 \mathrm{mg}(0.392 \mathrm{mmol})$ ammonium persulfate in $10 \mathrm{~mL}$ nanopure water. The reaction mixture was allowed to stir for $12 \mathrm{~h}$ for the polymerization. The polymer solution was transferred to dialysis tubes (MWCO $3500 \mathrm{Da}$ ) for dialysis for three days and lyophilized, obtaining a white solid powder with an average yield of $87 \% .{ }^{1} \mathrm{H}$ NMR $\left(\mathrm{D}_{2} \mathrm{O}, \mathrm{ppm}\right): \delta 3.14$ (s, CONHCH2CH2), 2.21 (s, CH2CH2CH2COO-), 1.99 (s, b, CONHCH2CH2), 1.59 (s, CH2CH2CH2COO-), 1.52 (s, CONHCH2CH2), 1.33 (s, CH2CH2CH2COO-), 1.70-1.25 (b, $\mathrm{CH} 2 \mathrm{CH} 2 \mathrm{CH} 2 \mathrm{CH} 2 \mathrm{CH} 2 \mathrm{CH} 2 \mathrm{CH} 2 \mathrm{CH} 2 \mathrm{CH} 2 \mathrm{COO}-, \mathrm{CH} 2 \mathrm{CHCO},) .{ }^{13} \mathrm{C} \mathrm{NMR}\left(\mathrm{D}_{2} \mathrm{O}, \mathrm{ppm}\right): \delta$ $183.57,176.09,39.58,37.74,37.54,28.99,28.29,26.87,26.33,26.08,25.85,25.57 . M_{n}=$ $82,300-170,600 . M_{w}=358,400-655,900$. IR: $3500-3000,2912,2843,1642,1552,1402$, 1302, $1101 \mathrm{~cm}-1$. DSC: $(\mathrm{Tg})=97.6^{\circ} \mathrm{C} .50: 50$ composition ratio of $\mathrm{P}\left(\mathrm{A}_{6 \mathrm{ACA}} \mathrm{A.5}^{-\mathrm{co}}\right.$ A11 $\mathrm{AUA}_{0.5}$ ) was the feeding ratio of the radical polymerization. 50:50 feeding ratio should be very close to the actual composition ratio of the resulting copolymer, based on the nearly quantitative conversion of two monomers after the polymerization.

\section{Preparation of enteric elastic polymer gels with various compositions (a typical procedure)}

To a well-mixed solution containing $1 \mathrm{~g}$ PA6ACA sodium salt, $0.853 \mathrm{~g}$ of poly(methacylic acid-co-ethyl acrylate) (EUDRAGIT ${ }^{\circledR} \mathrm{L} 100-55$ ) and $0.183 \mathrm{~g} \mathrm{NaOH}$ dissolved in $45 \mathrm{~mL}$ nanopure water, a solution of $5 \mathrm{~mL}$ of $6 \mathrm{M} \mathrm{HCl}$ (diluted from ACS grade concentrated 37\% $\mathrm{HCl})$ was quickly added. The mixture was put on the vortex shaker for $5 \mathrm{~min}$, then transferred into thick-wall centrifuge tubes (Beckman Coulter Inc.) and centrifuged in a Beckman Coulter Ultracentrifuge (Avanti ${ }^{\circledR} \mathrm{J}-26 \mathrm{XP}$ ) using an SW 32 Ti rotor at 32,000 rpm for $2 \mathrm{~h}$ at $20^{\circ} \mathrm{C}$. The resulting enteric elastic polymer gels with PA6ACA/L100-55 ratio 1:1 were extracted from the bottom of the ultracentrifuge tube. 


\section{Nunclear Magnetic Resonance (NMR) Characterization}

${ }^{1} \mathrm{H}$ NMR and ${ }^{13} \mathrm{C}$ NMR spectra were recorded on VARIAN Mercury 300 NMR SPECTROMETER with an Oxford Instruments Ltd. superconducting magnet - A $300 \mathrm{MHz}$ NMR spectrometer interfaced to a UNIX computer using VNMR 6.1c software. Chemical shifts were referenced to the solvent resonance signals.

\section{Gel Permeation Chromatography}

Aqueous gel permeation chromatography (GPC) was conducted on a Viscotek system (Malvern) equipped with an isocratic pump Viscoteck VE 1122 solvent delivery system, TDA 305 triple detector array, and 3x TSK Gel GMPWxL column with Guard column. The system was equilibrated at $30{ }^{\circ} \mathrm{C}$ in pre-filtered water containing $0.05 \mathrm{M} \mathrm{NaNO}_{3}$ at the flow rate set to $1.00 \mathrm{~mL} / \mathrm{min}$ ). Polymer solutions were prepared at a concentration of ca. 1-3 $\mathrm{mg} / \mathrm{mL}$ and an injection volume of $200 \mu \mathrm{L}$ was used. Data collection and analysis were performed with ChemStation for LC (Agilent) and OmniSEC v. 4,6,1,354 software (Malvern). The system was calibrated with poly(ethylene oxide) standards (Sigma) ranging from 400 to $511,000 \mathrm{Da}\left(M_{p}\right)$. The charged synthetic polymers in this manuscript may interact with the GPC column even when the salt eluent is used, therefore the GPC molecular weights based on uncharged PEO standards may be an underestimate. ${ }^{44}$

\section{Infrared Spectroscopy (IR)}

IR spectra were recorded on an ALPHA FT-IR Spectrometer (Bruker Corp.) and analyzed using OPUS v. 6,5,92 software.

Differential scanning calorimetry (DSC). Glass transition temperatures ( $\mathrm{Tg}$ ) were measured by differential scanning calorimetry on a PerkinElmer DSC 8000 (PerkinElmer, Inc.), with heating rate and cooling rate of $5{ }^{\circ} \mathrm{C} / \mathrm{min}$ in the range of $-50{ }^{\circ} \mathrm{C}$ and $150{ }^{\circ} \mathrm{C}$ under nitrogen atmosphere. Measurements were analyzed using Pyris v 11.0.0.0449 software. The Tg was taken as the midpoint of the inflection tangent, upon the third heating scan of three heating/ cooling cycles.

\section{Water content measurement and SEM analysis}

Three formulations were dried by lyophilization for 48 hours to measure their water content. The morphology of lyophilized polymer gels was analyzed by using a Zeiss Ultra55 field emission scanning electron microscope after being carbon coated.

\section{Small-angle X-ray Scattering (SAXS)}

SAXS experiments were conducted by DND-CAT of the Advanced Photon Source at Argonne National Laboratory. X-rays of wavelength $\lambda=0.73 \AA$ were used and each measurement was performed at room temperature using three different sample-to-detector distances $(0.2,1.0$, and $7.5 \mathrm{~m})$ to cover an q-range of $0.0026<\mathrm{q}<4.4 \AA$ - 1 , where $\mathrm{q}=(4 \pi / \lambda)$ $\sin (\theta / 2)$ is the magnitude of the scattering vector and $\theta$ is the scattering angle. Our gel samples were prepared into a disk shape and fixed vertically to have the x-ray beam pass through the center of the wet samples. Samples were approximately $1.0 \mathrm{~mm}$ thick and $3.0 \mathrm{~cm}$ in diameter. 


\section{Immersion tensile testing}

MTS Synergie 400 Tensile Test Machine equipped with a circulating and heating Bionix Mini Bath and an electronic temperature probe was used for the immersion tensile testing. For testing, EEs were cut in about $2 \mathrm{~mm} \times 2 \mathrm{~mm} \times 20 \mathrm{~mm}$ pieces, and held by wedge action grips, exposing 6-12 mm for the testing. SGF at $37{ }^{\circ} \mathrm{C}$ was added into the bath and EEs were allowed to equilibrate in SGF for $10 \mathrm{~min}$ before pulling. The stretch rate was set to 10 $\mathrm{mm} \cdot \mathrm{min}-1$. EEs were submerged in SGF during the whole testing process until the fracture.

\section{Dissolution studies}

EEs were cut into $\sim 1 \mathrm{~cm}^{3}$ sized cubes and submerged in either $40 \mathrm{~mL}$ SGF or SIF in a 50 $\mathrm{mL}$ VWR centrifuge tube. 6 replicates for each time point and condition were incubated at $37{ }^{\circ} \mathrm{C}$ on a shaker plate at $250 \mathrm{rpm}$. The solutions were exchanged with fresh SGF or SIF every 12 hours. At each time point, cubes were lyophilized for 48 hours before weighing. The remaining mass percentage equals the ratio of remaining dried weight to initial dried weight.

\section{Swelling tests}

Pre-weighted EEs cubes $\left(\sim 1 \mathrm{~cm}^{3}\right)$ were submerged in either $40 \mathrm{~mL}$ SGF blending with certain ratio of vegetable oil $(10 \%)$ or ethanol $(10-50 \%)$ in a $50 \mathrm{~mL}$ VWR centrifuge tube. 3 replicates for each solvent condition were incubated at $37{ }^{\circ} \mathrm{C}$ on a shaker plate at $250 \mathrm{rpm}$. After 24 hours, samples were weighted and compared with initial weights. For SGF with $10 \%$ vegetable oil, EE didn't gain detectable weight. For SGF with ethanol, swelling data is shown in Fig. S4.

\section{Cytotoxicity study}

PA6ACA sodium salt and L100-55 were dissolved in an aqueous $\mathrm{NaOH}$ solution.

Subsequently the $\mathrm{pH}$ was adjusted to 7.0 using $1 \mathrm{M} \mathrm{HCl}$. The final polymer solution was diluted with Dulbecco's Modified Eagle Medium (DMEM) (Life Technologies) to 100 $\mathrm{mg} / \mathrm{mL}$ before testing. Cytotoxicity was tested on HeLa, HEK293, C2BBe1 (ATCC) and HT29-MTX-E12 cells (Public Health England) by seeding them in a 96-well plate at a density of $6 \times 103,16 \times 103,16 \times 103$ and $2 \times 104$ cells/well respectively. HeLa and HEK293 cells were cultured in $100 \mu \mathrm{L}$ DMEM containing $1 \%$ non-essential amino acids, $10 \%$ fetal bovine serum (FBS) and 1\% penicillin-streptomycin solution (Life Technologies) per well. C2BBe1 and HT29-MTX-E12 cells were cultured in the same medium but was additionally supplemented with $4 \mathrm{mg} / \mathrm{mL}$ human transferrin (Life Technologies). Cells were kept in culture for 3 days before replacing the medium, to which the dissolved aqueous polymer solutions were added (final concentrations of polymers ranged from $0.078-20 \mathrm{mg}$ / $\mathrm{mL}$ ). After $72 \mathrm{~h}$, cytotoxicity was quantified by adding $10 \mu \mathrm{L}$ alamarBlue reagent (Life Technologies) to each well. The contents were mixed well and then allowed to incubate at $37^{\circ} \mathrm{C}$ for $1 \mathrm{~h}$. Absorbance at $570 \mathrm{~nm}$ was recorded on an Infinite M200Pro (Tecan) using $600 \mathrm{~nm}$ as reference wavelength. A positive control was provided by lysing cells with $1 \%$ Tween-20 and cells that were not subject to any polymer provided a negative control. Cell viability was calculated by the following equation: Cell viability $(\%)=100 \times$ 
(Absorbance(sample)-Absorbance(positive control)) / (Absorbance(negative control)Absorbance(positive control)).

\section{Construction of gastric retentive devices bearing EE and polycaprolactone (PCL)}

An Objet 3D printer using DurusWhite RGD430 build material and Support Fullcure 705 as support material was used to generate shapes as positive models. Negative molds were created by casting polydimethylsiloxane (PDMS) (SYLGARD® 184 SILICONE ELASTOMER KIT, Dow Corning) around positive models. EE (PA6ACA/L100-55 1:2 ratio) was cut into cubes or cuboids to fit into the PDMS molds and dried by vacuum. Beads of PCL (Sigma, $M_{n} 80 \mathrm{k}$ ) were placed between EE pieces in the PDMS molds and melted at $70{ }^{\circ} \mathrm{C}$ for 12 hours before cooling to room temperature. Resulting devices bearing EE and PCL were submerged in SGF for 2 days to completely hydrate EE before devices were removed from the molds.

\section{Pig Large animal model}

All procedures were conducted in accordance with protocols approved by the Massachusetts Institute of Technology Committee on Animal Care. Six separate female Yorkshire pigs weighing approximately $45-55 \mathrm{~kg}$ were used for in vivo evaluation. Prior to the procedures the animals were fasted overnight. On the day of the procedure, the morning feed was held and the animals were sedated with Telazol (tiletamine/zolazepam) $5 \mathrm{mg} / \mathrm{kg}$, xylazine 2 $\mathrm{mg} / \mathrm{kg}$, and atropine $0.04 \mathrm{mg} / \mathrm{kg}$. To ensure gastric placement of the devices the devices were placed in the stomach with the use of an esophageal overtube (US Endoscopy, Mentor, Ohio) which was placed endoscopically in the esophagus. Radiographs were performed every 48-72 hours to monitor the integrity and transit of the devices as well as any radiographic evidence of bowel obstruction or perforation. Furthermore all animals were monitored clinically at least twice a day for any evidence of obstruction including poor feeding, poor defecation, abdominal distension and vomiting. Where radio-opaque fiducials were omitted from prototype devices visualization was performed endoscopically.

\section{Supplementary Material}

Refer to Web version on PubMed Central for supplementary material.

\section{Acknowledgement}

This work was sponsored by Mr. Bill Gates and funded in part by Bill and Melinda Gates Foundation Grant OPP1096734 (to R.L.) and NIH Grant EB000244 (to R.L.). The paper was partly sponsored by the Alexander von Humboldt Foundation under the auspices of the Max Planck Research Award to R.L. funded by the Federal Ministry of Education and Research. A.M.B. was supported in part by NIH T32 5T32HL007604-29. J.Z. was supported by the Laboratory Directed Research and Development program at Oak Ridge National Laboratory, which is sponsored by the Scientific User Facilities Division, Office of Basic Energy Sciences, U.S. Department of Energy. Use of the Advanced Photon Source, an Office of Science User Facility operated for the U.S. Department of Energy (DOE) Office of Science by Argonne National Laboratory, was supported by the U.S. DOE under Contract No. DE-AC02-06CH11357. We would like to thank Dr. J. Haupt and Ms. M. Jamiel for expert veterinary support. We are indebted to Dr. Lowell Wood, Dr. Philip Eckhoff, Dr. Dan Hartman, Dr. Steven Kern, Dr. Susan Hershenson, Dr. Boris Nikolic and Mr. Bill Gates for fruitful discussions stimulating the development of this material. 


\section{References}

1. Kethu SR, et al. Endoluminal bariatric techniques. Gastrointestinal Endoscopy. 2012; 76:1-7. doi:DOI 10.1016/j.gie.2012.02.020. [PubMed: 22579259]

2. Genco A, et al. BioEnterics Intragastric Balloon: The Italian experience with 2,515 patients. Obes Surg. 2005; 15:1161-1164. doi:Doi 10.1381/0960892055002202. [PubMed: 16197790]

3. Won YW, et al. Oligopeptide complex for targeted non-viral gene delivery to adipocytes. Nat Mater. 2014; 13:1157-1164. doi:Doi 10.1038/Nmat4092. [PubMed: 25282508]

4. Tao H, et al. Silk-Based Conformal, Adhesive, Edible Food Sensors. Adv Mater. 2012; 24:10671072. doi:DOI 10.1002/adma.201103814. [PubMed: 22266768]

5. Kim YJ, Wu W, Chun SE, Whitacre JF, Bettinger CJ. Biologically derived melanin electrodes in aqueous sodium-ion energy storage devices. P Natl Acad Sci USA. 2013; 110:20912-20917. doi:DOI 10.1073/pnas.1314345110.

6. Byrne C, Lim CL. The ingestible telemetric body core temperature sensor: a review of validity and exercise applications. Brit J Sport Med. 2007; 41:126-133. doi:DOI 10.1136/bjsm.2006.026344.

7. Belknap R, et al. Feasibility of an Ingestible Sensor-Based System for Monitoring Adherence to Tuberculosis Therapy. Plos One. 2013 doi:ARTN e53373 DOI 10.1371/journal.pone.0053373.

8. Moes AJ. Gastroretentive Dosage Forms. Crit Rev Ther Drug. 1993; 10:143-195.

9. Hwang SJ, Park H, Park K. Gastric retentive drug-delivery systems. Crit Rev Ther Drug. 1998; 15:243-284.

10. Singh BN, Kim KH. Floating drug delivery systems: an approach to oral controlled drug delivery via gastric retention. J Control Release. 2000; 63:235-259. doi:Doi 10.1016/ S0168-3659(99)00204-7. [PubMed: 10601721]

11. Fuhrmann G, et al. Sustained gastrointestinal activity of dendronized polymer-enzyme conjugates. Nat Chem. 2013; 5:582-589. doi:Doi 10.1038/Nchem.1675. [PubMed: 23787748]

12. Laulicht B, Gidmark NJ, Tripathi A, Mathiowitz E. Localization of magnetic pills. Proceedings of the National Academy of Sciences. 2011; 108:2252-2257. doi:10.1073/pnas.1016367108.

13. Salessiotis N. Measurement of the diameter of the pylorus in man: Part I. Experimental project for clinical application. The American Journal of Surgery. 1972; 124:331-333. doi:http://dx.doi.org/ 10.1016/0002-9610(72)90036-0. [PubMed: 5056893]

14. Munk, JF.; Gannaway, RM.; Hoare, M.; Johnson, AG. Gastrointestinal Motility in Health and Disease. Duthie, HL., editor. Springer; Netherlands: 1978. p. 349-359.Ch. 38

15. Sultan M, Norton R. Esophageal diameter and the treatment of achalasia. Digest Dis Sci. 1969; 14:611-618. doi:10.1007/BF02239272.

16. Vanstiegmann G, Cambre T, Sun JH. A New Endoscopic Elastic Band Ligating Device. Gastrointestinal Endoscopy. 1986; 32:230-233. doi:Doi 10.1016/S0016-5107(86)71815-4. [PubMed: 3487484]

17. Dumonceau JM. Evidence-based Review of the Bioenterics Intragastric Balloon for Weight Loss. Obes Surg. 2008; 18:1611-1617. doi:DOI 10.1007/s11695-008-9593-9. [PubMed: 18568377]

18. Cheifetz AS, et al. The Risk of Retention of the Capsule Endoscope in Patients with Known or Suspected Crohn's Disease. Am J Gastroenterol. 2006; 101:2218-2222. [PubMed: 16848804]

19. McGovern R, Barkin JS, Goldberg RI, Phillips RS. Duodenal obstruction: a complication of percutaneous endoscopic gastrostomy tube migration. The American journal of gastroenterology. 1990; 85:1037-1038. [PubMed: 2197857]

20. Trande $P$, et al. Efficacy, tolerance and safety of new intragastric air-filled balloon (Heliosphere BAG) for obesity: the experience of 17 cases. Obes Surg. 2010; 20:1227-1230. doi:10.1007/ s11695-008-9786-2. [PubMed: 19082675]

21. Roman S, et al. Intragastric Balloon for "Non-Morbid" Obesity: A Retrospective Evaluation of Tolerance and Efficacy. Obes Surg. 2004; 14:539-544. doi:10.1381/096089204323013587. [PubMed: 15130235]

22. Traverso G, Langer R. Perspective: Special delivery for the gut. Nature. 2015; 519:S19-S19. doi: 10.1038/519S19a. [PubMed: 25806494] 
23. Lappas LC, Mckeehan W. Synthetic Polymers as Potential Enteric and Sustained-Release Coatings. Journal of Pharmaceutical Sciences. 1962; 51:808. doi:DOI 10.1002/jps.2600510828. [PubMed: 14462360]

24. Siepmann F, Siepmann J, Walther M, MacRae RJ, Bodmeier R. Polymer blends for controlled release coatings. J Control Release. 2008; 125:1-15. doi:DOI 10.1016/j.conrel.2007.09.012. [PubMed: 18022722]

25. Yan XZ, Wang F, Zheng B, Huang FH. Stimuli-responsive supramolecular polymeric materials. Chem Soc Rev. 2012; 41:6042-6065. doi:Doi 10.1039/C2cs35091b. [PubMed: 22618080]

26. Wojtecki RJ, Meador MA, Rowan SJ. Using the dynamic bond to access macroscopically responsive structurally dynamic polymers. Nat Mater. 2011; 10:14-27. doi:Doi 10.1038/ Nmat2891. [PubMed: 21157495]

27. Li JH, Viveros JA, Wrue MH, Anthamatten M. Shape-memory effects in polymer networks containing reversibly associating side-groups. Adv Mater. 2007; 19:2851. doi:DOI 10.1002/adma. 200602260.

28. Yan XZ, et al. A Multiresponsive, Shape-Persistent, and Elastic Supramolecular Polymer Network Gel Constructed by Orthogonal Self-Assembly. Adv Mater. 2012:362. doi:DOI 10.1002/adma. 201103220. [PubMed: 22161963]

29. Jang SG, Kramer EJ, Hawker CJ. Controlled Supramolecular Assembly of Micelle-Like Gold Nanoparticles in PS-b-P2VP Diblock Copolymers via Hydrogen Bonding. J Am Chem Soc. 2011; 133:16986-16996. doi:Doi 10.1021/Ja206615c. [PubMed: 21923144]

30. Tee BCK, Wang C, Allen R, Bao ZN. An electrically and mechanically self-healing composite with pressure- and flexion-sensitive properties for electronic skin applications. Nat Nanotechnol. 2012; 7:825-832. doi:Doi 10.1038/Nnano.2012.192. [PubMed: 23142944]

31. Chen YL, Kushner AM, Williams GA, Guan ZB. Multiphase design of autonomic self-healing thermoplastic elastomers. Nat Chem. 2012; 4:467-472. doi:Doi 10.1038/Nchem.1314. [PubMed: 22614381]

32. Schaaf P, Schlenoff JB. Saloplastics: Processing Compact Polyelectrolyte Complexes. Adv Mater. 2015 n/a-n/a, doi:10.1002/adma.201500176.

33. Lendlein A, Neffe AT, Jérôme C. Advanced Functional Polymers for Medicine. Advanced Healthcare Materials. 2014; 3:1939-1940. doi:10.1002/adhm.201400718. [PubMed: 25491097]

34. Stuart MAC, et al. Emerging applications of stimuli-responsive polymer materials. Nat Mater. 2010; 9:101-113. doi:Doi 10.1038/Nmat2614. [PubMed: 20094081]

35. Sathish D, Himabindu S, Kumar YS, Shayeda, Rao YM. Floating Drug Delivery Systems for Prolonging Gastric Residence Time: A Review. Curr Drug Deliv. 2011; 8:494-510. [PubMed: 21696354]

36. Phadke A, et al. Rapid self-healing hydrogels. Proceedings of the National Academy of Sciences. 2012; 109:4383-4388. doi:10.1073/pnas.1201122109.

37. Luzar A, Chandler D. Structure and hydrogen bond dynamics of water-dimethyl sulfoxide mixtures by computer simulations. The Journal of Chemical Physics. 1993; 98:8160-8173. doi:doi:http://dx.doi.org/10.1063/1.464521.

38. Woodruff MA, Hutmacher DW. The return of a forgotten polymer-Polycaprolactone in the $21 \mathrm{st}$ century. Prog Polym Sci. 2010; 35:1217-1256. doi:DOI 10.1016/j.progpolymsci.2010.04.002.

39. Kearney CJ, Mooney DJ. Macroscale delivery systems for molecular and cellular payloads. Nat Mater. 2013; 12:1004-1017. doi:Doi 10.1038/Nmat3758. [PubMed: 24150418]

40. Khosla R, Davis SS. The effect of tablet size on the gastric emptying of non-disintegrating tablets. International Journal of Pharmaceutics. 1990; 62:R9-R11. doi:http://dx.doi.org/ 10.1016/0378-5173(90)90243-W.

41. Cargill R, et al. Controlled Gastric Emptying. 1. Effects of Physical Properties on Gastric Residence Times of Nondisintegrating Geometric Shapes in Beagle Dogs. Pharm Res. 1988; 5:533-536. doi:10.1023/A:1015981627525. [PubMed: 3244664]

42. Martinez MN, Papich MG. Factors influencing the gastric residence of dosage forms in dogs. Journal of Pharmaceutical Sciences. 2009; 98:844-860. doi:10.1002/jps.21499. [PubMed: 18661535] 
43. Swindle MM, Makin A, Herron AJ, Clubb FJ, Frazier KS. Swine as Models in Biomedical Research and Toxicology Testing. Vet Pathol. 2012; 49:344-356. doi:Doi 10.1177/0300985811402846. [PubMed: 21441112]

44. Cooper AR, Matzinger DP. Aqueous gel permeation chromatography: The effect of solvent ionic strength. Journal of Applied Polymer Science. 1979; 23:419-427. doi:10.1002/app. 1979.070230211. 
a

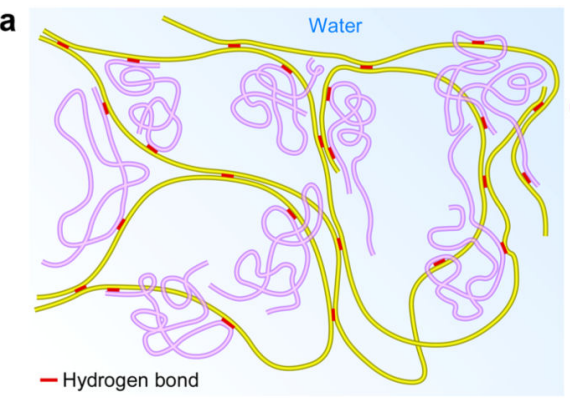

b

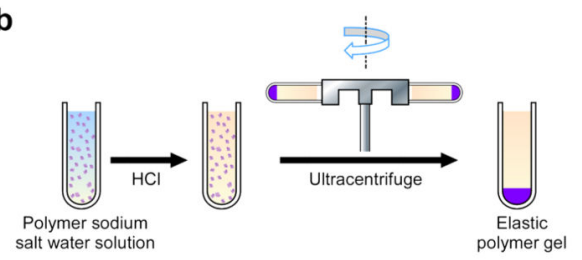

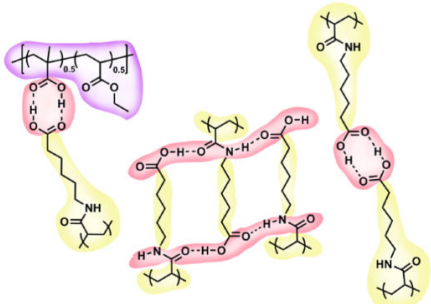

C

d

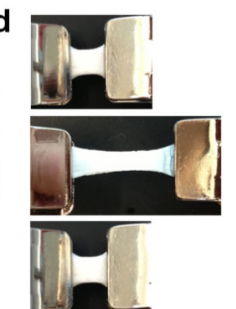

Figure 1.

Schematic representation of the enteric elastomer including manufacturing and macroscopic characteristics. a, Proposed supramolecular polymer gel network. Structures in yellow, synthesized poly(acryloyl-6-aminocaproic acid) (PA6ACA, $M_{n}=61,600-112,700 . M_{w}=$ 347,300 - 466,300); structures in purple, linear poly(methacrylic acid-co-ethyl acrylate) (EUDRAGIT® L 100-55, $M_{n}=72,300 . M_{w}=241,000$ ); red part, inter-polymer hydrogen bonds. b, Manufacturing process of the polymer gel. Left, the homogeneous solution of PA6ACA sodium salt solution and L100-55 sodium salt solution with varying polymer weight ratios. Middle, the addition of $\mathrm{HCl}$ solution resulting precipitation. Right, formation of the elastic polymer gel after ultracentrifugation. c, Photo of a piece of the polymer gel obtained after ultracentrifugation. $d$, Images of stretch and recovery testing of a polymer gel with PA6ACA:L100-55 = 1:2. Top, $1.5 \mathrm{~cm}$ piece of polymer gel held between two clamps. Middle, stretching of the polymer gel to 3 times initial length. Bottom, recovery of polymer gel $5 \mathrm{~min}$ after the external force was removed. Scale bar is $2 \mathrm{~cm}$ for $\mathrm{c}$ and $\mathrm{d}$. 

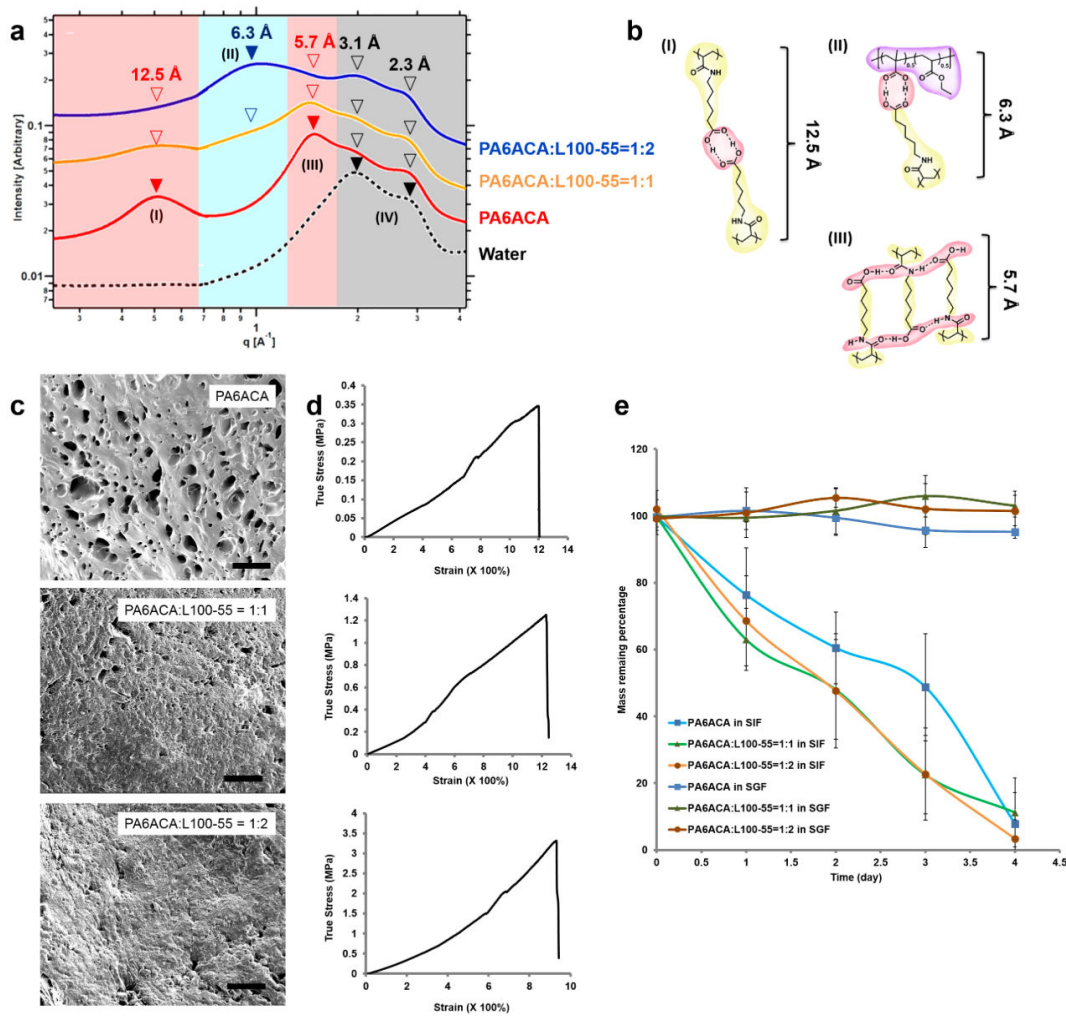

Figure 2.

Physical characterization of the enteric elastomer. a, SAXS data of EEs identified three major hydrogen-bonding structures. b, The carboxyl groups on PA6ACA interacting with the opposing carboxyl groups on PA6ACA in a face-on configuration (I), the carboxyl groups within PA6ACA interacting with the carboxyl groups on L100-55 in a face-on configuration (I), and the carboxyl groups on PA6ACA interacting with amide groups of the opposing pendant side chain in an interleaved configuration (III). c, SEM images of dried polymer gels with various PA6ACA/L100-55 weight ratios (top 1:0, middle 1:1, bottom 1:2). Scale bars $50 \mu \mathrm{m}$. d, True stress-strain plots of polymer gels with PA6ACA/L100-55 weight ratios (top 1:0, middle 1:1, bottom 1:2) stretched to breaking at $10 \mathrm{~mm} \cdot \mathrm{min}^{-1}$. e, Dissolution study of polymer gels in simulated gastric fluid (SGF) and simulated intestinal fluid (SIF) showing complete dissolution of the polymer gels in SIF for about 4 days and no significant mass loss in SGF for the same period of time. EEs in $\sim 1 \mathrm{~cm}^{3}$ size were incubated in SGF and SIF at $37^{\circ} \mathrm{C}$, and then lyophilized. The mass remaining percentage equals the ratio of remaining dried weights to initial dried weights. The vertical error bars correspond to the standard deviations of total 6 samples per formulation. 
a
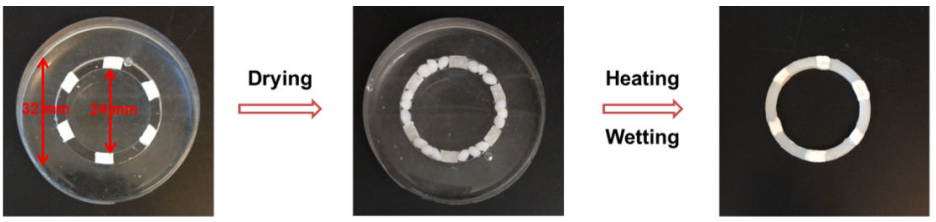

b
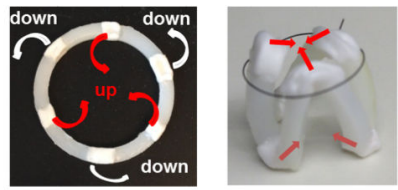

c
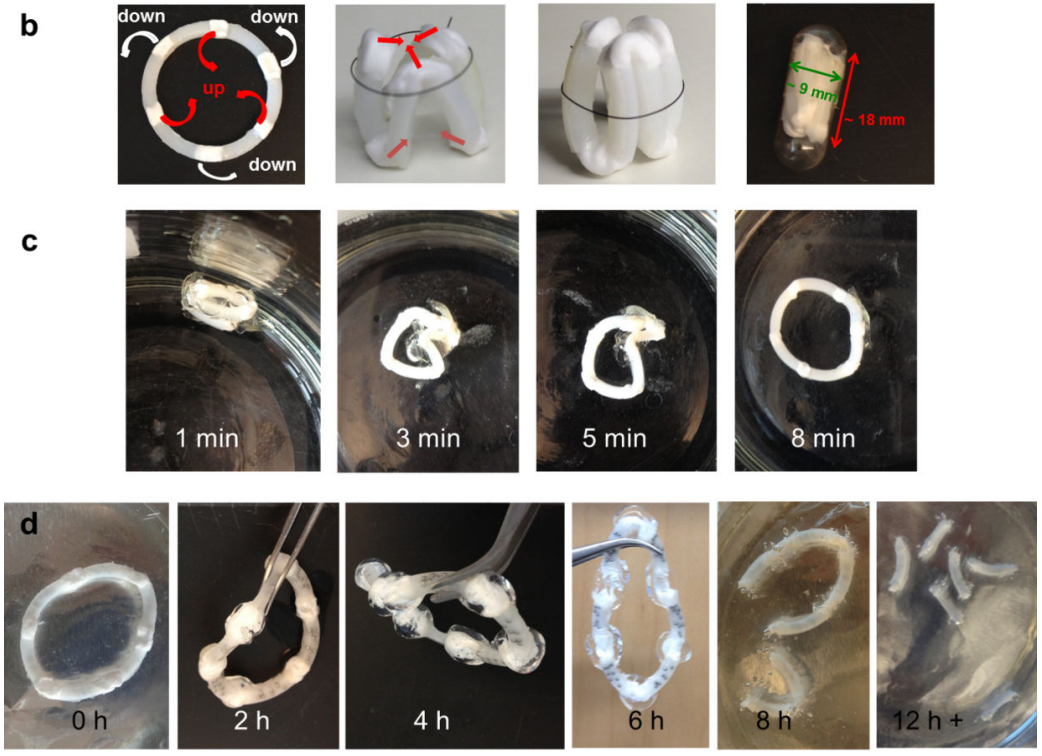

Figure 3.

Construction of a ring-shaped gastric retentive device and in vitro testing of its elasticity and enteric property. a, Construction of a circle composed of polycaprolactone (PCL) arcs with intervening EE polymer gel linkers by first fitting cubic polymer gels and PCL beads alternatively in a circle-shaped polydimethylsiloxane (PDMS) mold followed by melting PCL at $70{ }^{\circ} \mathrm{C}$. b, Folding of the ring into a standard gelatin 000 capsule by utilizing the elasticity of the polymer gel. c, Escape from the capsule and recovery to the ring shape after dissolution of the gelatin capsule in SGF at $37^{\circ} \mathrm{C}$. d, Progressive dissolution of EE supramolecular polymer gel linkers in SIF at $37{ }^{\circ} \mathrm{C}$ for the dissociation of the circle-shaped device into PCL arcs by utilizing the enteric property of the polymer gel. 

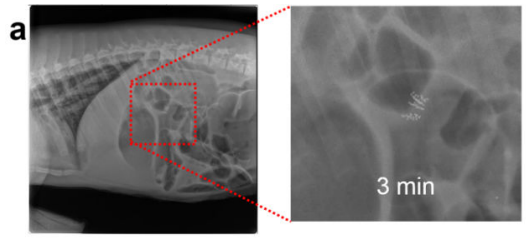

b
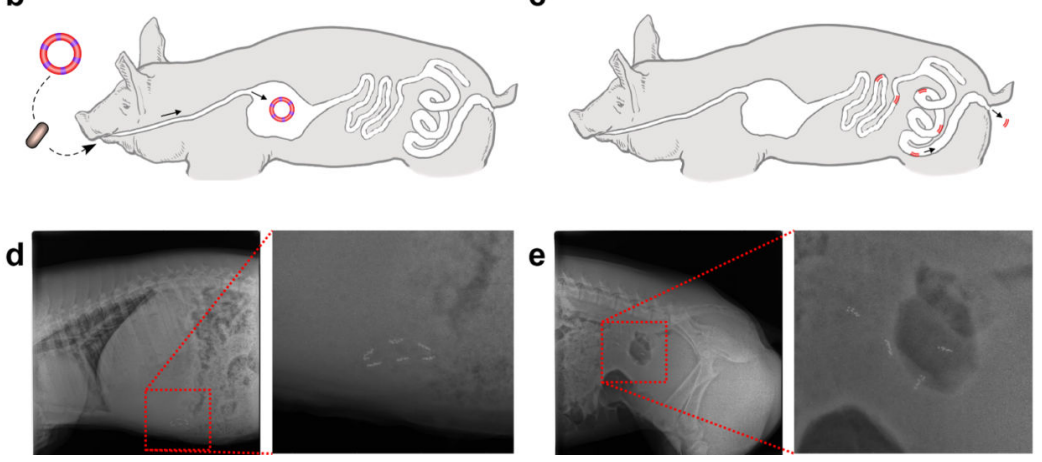

Figure 4.

In vivo evaluation of the ring-shaped devices in Yorkshire pigs. a, Recovery of the ring shape after delivery of an encapsulated ring-shaped device through the esophagus and dissolution of the gelatin capsule in stomach. b, Schematic representation of the delivery and gastric retention of a ring-shaped device. c, Schematic illustration of safe passage of PCL arcs through the small and large intestine upon dissociation of the ring-shaped device as the result of dissolution or partial dissolution of EE linkers. $d$, X-ray image of a ring-shaped device residing in the gastric cavity of a Yorkshire pig. e, X-ray image of four PCL arcs passing through the intestine after dissolution of the EE linker. For visualization purposes, 6-10 radio-opaque stainless steel beads ( $1 \mathrm{~mm}$ diameter) were incorporated in every PCL arm. Total bead mass was $\sim 200 \mathrm{mg}$, while the whole device (with iron beads) weight was $\sim 1000 \mathrm{mg}$. 


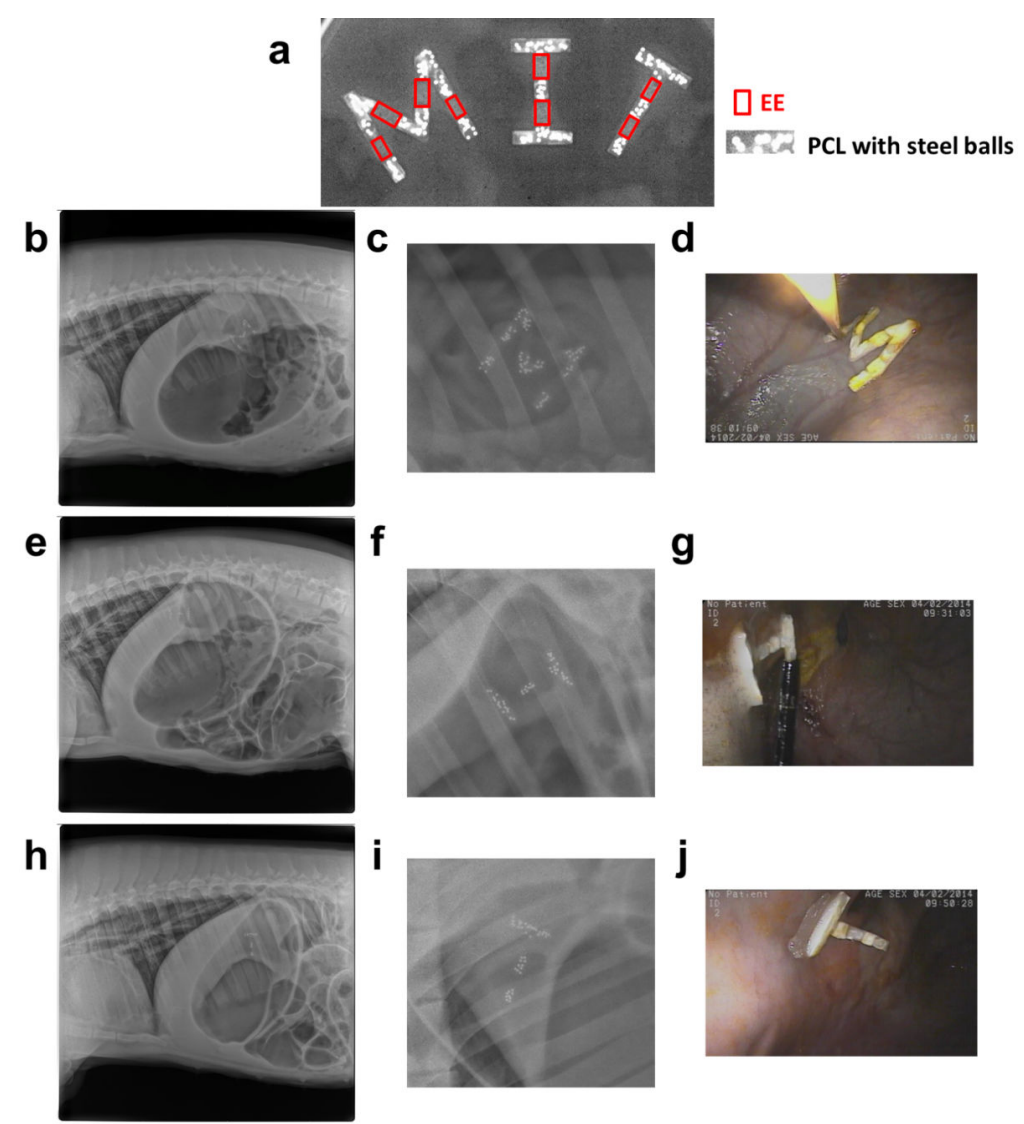

Figure 5.

In vivo evaluation of M- $(30 \mathrm{~mm} \times 30 \mathrm{~mm} \times 4 \mathrm{~mm})$, I- $(30 \mathrm{~mm} \times 18 \mathrm{~mm} \times 4 \mathrm{~mm})$, and Tshaped (30 $\mathrm{mm} \times 18 \mathrm{~mm} \times 4 \mathrm{~mm}$ ) devices. A. X-ray image of M-, I-, and T-shaped devices made of EE as joints and PCL with embedded steel beads as X-ray contrast agent. B-D. Xray images (b and c) and endoscopic image (d) showing gastric retention of M-shaped device. E-G. X-ray images (e and f) and endoscopic image (g) showing gastric retention of I-shaped device. H-J. X-ray images (h and i) and endoscopic image (i) showing gastric retention of $\mathrm{T}$-shaped device. 